\title{
Legionella pneumophila serogroup 3 pneumonia in a patient with low-grade 4 non-Hodgkin lymphoma: a case report
}

\author{
Antonella Mencacci ${ }^{1}$, Cristina Corbucci ${ }^{1}$, Alessio Castellani ${ }^{2}$, Paolo Furno ${ }^{2}$, Francesco Bistoni ${ }^{1}$ and \\ Anna Vecchiarelli $i^{*}$
}

\begin{abstract}
Introduction: Nosocomial legionellosis has generally been described in immunodepressed patients, but Legionella pneumophila serogroup 3 has rarely been identified as the causative agent.

Case presentation: We report the case of nosocomial L. pneumophila serogroup 3 pneumonia in a 70-year-old Caucasian man with non-Hodgkin lymphoma. Diagnosis was carried out by culture and real-time polymerase chain reaction of bronchoalveolar lavage fluid. The results of a urinary antigen test were negative. A hospital environmental investigation revealed that the hospital water system was highly colonized by L. pneumophila serogroups 3, 4, and 8 . The hospital team involved in the prevention of infections was informed, long-term control measures to reduce the environmental bacterial load were adopted, and clinical monitoring of legionellosis occurrence in high-risk patients was performed. No further cases of Legionella pneumonia have been observed so far.

Conclusions: In this report, we describe a case of legionellosis caused by L. pneumophila serogroup 3, which is not usually a causative agent of nosocomial infection. Our research confirms the importance of carrying out cultures of respiratory secretions to diagnose legionellosis and highlights the limited value of the urinary antigen test for hospital infections, especially in immunocompromised patients. It also indicates that, to reduce the bacterial load and prevent nosocomial legionellosis, appropriate control measures should be implemented with systematic monitoring of hospital water systems.
\end{abstract}

\section{Introduction}

Legionnaires' disease is often a hospital-acquired infection. In Italy, 9.2\% of nosocomial cases were recorded in 2009 with a fatality rate (34\%) significantly higher than community-acquired cases (12\%) [1]. This difference is due to the patients' state of immunodeficiency, which is known to be an important risk factor in contracting Legionella pneumonia. Often, hospital water systems are colonized by Legionella and this contamination is responsible for most cases of hospital-acquired legionellosis [2]. A link between the presence of the bacterium in hospital water systems and nosocomial legionellosis

\footnotetext{
* Correspondence: vecchiar@unipg.it

'Department of Experimental Medicine and Biochemical Sciences, Microbiology Section, University of Perugia, Via del Giochetto, Perugia 06122, Italy

Full list of author information is available at the end of the article
}

has been reported [3], suggesting that it is necessary to sample hospital water routinely for Legionella and make sure that this microorganism is not present in transplant units or other wards with significantly immunosuppressed patients [4]. Sixteen serogroups of Legionella pneumophila are known. Serogroup 1 is the most common in clinical and environmental isolates [5], whereas L. pneumophila serogroup 3 has rarely been isolated in immunocompromised patients [6].

\section{Case presentation}

In this report, a case of hospital-acquired pulmonary legionellosis in an immunocompromised patient - a 70year-old Caucasian man - is described. Sixteen years before he presented to us, he had a condition that was diagnosed as low-grade non-Hodgkin lymphoma of the B-cell small lymphocytic type (stage IV with mediastinal, 
abdominal, bone marrow, and superficial node site involvement), and nine courses of combined polychemotherapy were administered with cyclophosphamide, doxorubicin, vincristine, and prednisone. Four years before he presented to us, lymphoma chemotherapy was started again with cyclophosphamide and fludarabine because of a progression of mesenteric involvement of the disease. This regimen was interrupted four months later because of toxicity, and 11 courses of alemtuzumab were administered thereafter in the Oncology and Hematology Clinic of our division.

Three years before he presented to us, he was discharged from our ward after a two-week hospitalization for fever and pancytopenia (first hospital admission of this report). After seven days, he was hospitalized again, in another ward of the same hospital, because of fever, dyspnea, and bowel movements with loose stools (second admission). A chest X-ray showed an inflammatory infiltrate at the base of his right lung, and another slight infiltration seemed to be spreading in the upper field of his left lung. The results of laboratory tests were unremarkable except for significant increases of erythrocyte sedimentation rate and $\mathrm{C}$-reactive protein, minimal increases of aspartate aminotransferase and alanine aminotransferase, and mild anemia. The results of blood cultures, a cytomegalovirus antigen test in serum, and a Clostridium difficile toxin stool test were negative. A course of empirical antibiotic therapy with piperacillin/ tazobactam plus ciprofloxacin was started. A rapid defervescence ensued, and our patient was discharged after an eight-day course of antibiotics. However, two days after discharge, fever recurred and he was hospitalized again in our ward to investigate a possible opportunistic respiratory infection (third admission). $\mathrm{He}$ appeared mildly ill and complained of pleuritic pain at the base of his right lung when breathing deeply; a lowgrade fever was present, but his respiratory rate $(20$ breaths per minute), heart rate ( 88 beats per minute), and blood pressure $(120 / 70 \mathrm{~mm} \mathrm{Hg})$ were normal; his arterial blood oxygen saturation was $96 \%$ while he was breathing room air. Inspiratory crackles were evident over his right lower lung field, but the results of the physical examination were otherwise normal. Blood cultures were requested in order to check for common pathogens as an empiric antibiotic therapy was started with piperacillin/tazobactam. However, several hours after admission, his fever increased to over $38^{\circ} \mathrm{C}$ and persisted for five days. Therefore, a chest computed tomography (CT) scan and a bronchoscopy test with bronchoalveolar lavage (BAL) were performed and, although our patient's general clinical condition remained stable and blood culture results were negative, beta-lactams were replaced with clarithromycin to provide antibacterial coverage against possible atypical respiratory pathogens. The chest CT scan showed evidence of an area of lung parenchymal consolidation in his right lower lobe with adjacent ground-glass opacities and micronodules. No lymphadenopathy was evident. A microbiological examination of BAL fluid was negative for common Gram-negative and Gram-positive pathogens, fungi, Nocardia spp., Mycobacterium tubercolosis, Pneumocystis jiroveci, cytomegalovirus, adenovirus, and respiratory syncytial virus, but Legionella was detected after four days of incubation. For culture, BAL fluid, undiluted or diluted (1:10) in trypticase soy broth, was plated on a buffered charcoal yeast extract (BCYE) medium with $0.1 \%$ alpha-ketoglutaric acid and on selective BCYE agar medium supplemented with glycine, vancomycin, polymyxin B, and cycloheximide (GVPC) (Becton Dickinson, Milan, Italy). Colonies were identified as $L$. pneumophila serogroup 3 by using the Dresden monoclonal antibody panel [7]. The results of real-time polymerase chain reaction (PCR) assays performed on DNA extracted from BAL fluid were also positive for Legionella spp. (Nanogen Advanced Diagnostic, Torino, Italy). The results of urinary antigen tests for L. pneumophila serogroup 1 (Binax, Portland, ME, USA) and non-serogroup 1 (Biotest, Dreieich, Germany) were negative.

A prompt clinical response was observed after clarithromycin treatment. Two days after the start of macrolide treatment, our patient was afebrile and had no chest pain and his condition was generally improving and remained so thereafter. A chest X-ray performed two weeks later showed a slight reduction of right lung opacity, and he was discharged after 20 days of hospital stay while on clarithromycin therapy. This treatment was continued for seven more days. A follow-up chest CT scan obtained five weeks later revealed an almost complete resolution of the previously documented right lung consolidation and ground-glass areas.

An environmental investigation was carried out after the isolation of the infectious agent. First of all, our patient's home water system was investigated for the presence of L. pneumophila, but this pathogen was found in neither the cold nor the hot water system (data not shown). Given that the incubation time for L. pneumophila is generally two to 10 days and that, six days after being discharged from his first hospitalization (for pancytopenia), our patient was admitted again with respiratory symptoms, we suspected that he had been exposed to the microorganism during the period spent in the hospital. To verify this hypothesis, an intensive environmental investigation was carried out on the hospital's hot and cold water systems. A microbiological analysis of water samples, collected from the sink and shower of our patient's room from the first hospital admission, revealed that the hot water sample was 
Table 1 Microbiological analysis of hot water sampled from our patient's room from his first admission

\begin{tabular}{lcc}
\hline $\begin{array}{l}\text { Sampling } \\
\text { site }\end{array}$ & $\begin{array}{c}\text { Sampling } \\
\text { mode }\end{array}$ & $\begin{array}{c}\text { Legionella pneumophila load, CFU/ } \\
\text { L }\end{array}$ \\
\hline Bathroom sink & Pre-flushing & $4 \times 10^{3}$ \\
& Post-flushing & $4.5 \times 10^{3}$ \\
Shower & Pre-flushing & $4 \times 10^{2}$ \\
& Post-flushing & $3.5 \times 10^{2}$ \\
\hline
\end{tabular}

CFU/L: colony-forming units per liter.

contaminated by L. pneumophila serogroup 3 (Table 1). The concentration of 8000 colony-forming units per liter (CFU/L) exceeded the $1000 \mathrm{CFU} / \mathrm{L}$ of the European guidelines for hotels and the $1000 \mathrm{CFU} / \mathrm{L}$ of the Italian guidelines for hospitals [8]. Also, a lot of other sites of the hospital's hot water system were highly colonized by L. pneumophila, including serogroups 3,4 , and 8 (Table 2 ). Legionella was not isolated from the cold water system. Molecular typing of L. pneumophila clinical and environmental isolates was carried out by using the amplified fragment length polymorphism method [9], and the genomic profile of the L. pneumophila clinical strain matched with that of the L. pneumophila serogroup 3 strain (data not shown).

On the basis of these results, an extensive program of microbiological controls in the entire hospital water system was implemented. In accordance with the European guidelines, shock hyperchlorination was applied to the water distribution system [10] with a single addition of chlorine to the water to obtain concentrations of free residual chlorine of 20 to $50 \mathrm{mg} / \mathrm{L}$ throughout the water system, including the distal point. After this treatment, cultures of water samples collected one day after hyperchlorination showed a decrease in L. pneumophila concentration. However, a considerable increase of CFU was observed one month later (Table 3). Therefore, continuous hyperchlorination treatment (free residual chlorine of 1 to $3 \mathrm{mg} / \mathrm{L}$ ) of the hospital water system is now routinely applied and monitoring is performed every six months. In addition, periodic monitoring of the Legionella CFU in the water system is carried out along with careful clinical surveillance of legionellosis cases in low- and high-risk patients with pneumonia. No other nosocomial Legionella pneumonia cases have been observed so far.

\section{Discussion}

Owing to the difficulty of distinguishing legionellosis from other forms of pneumonia by clinical and roentgenographic analysis, specific laboratory diagnostics should be enforced in order to increase the detection rate of nosocomial Legionnaires' disease. Culture remains the most specific diagnostic procedure for legionellosis [5]. The usefulness of urinary antigen detection for the diagnosis of Legionnaires' disease is well documented. However, because it is fast and easy to perform, this test has caused a decrease in the use of cultures to detect infection, resulting in incomplete surveillance for legionellosis [5]. The Binax urinary antigen kit detects L. pneumophila serogroup 1 antigen [11], whereas the Biotest urine antigen enzyme immunoassay has a wide range of cross-reactivity to the other serogroups and species. Benson and colleagues [12] reported that both Binax and Biotest urinary antigen kits were capable of detecting multiple serogroups of $L$. pneumophila, including serogroup 3. However, in this case and in other legionellosis cases [6], the urine antigen test was negative when the above-mentioned kits were used and the correct diagnosis was made only when BAL fluid-specific culture and real-time PCR methods were used. This demonstrates the importance of using at least one of these methods as part of the routine microbiological testing of BAL fluid, especially in immunocompromised patients with pulmonary

Table 2 Microbiological analysis of hot water sampled from the entire water system of the hospital

\begin{tabular}{lcc}
\hline Sampling site & Sampling mode & Legionella pneumophila load, CFU/L \\
\hline Water mains & Pre-flushing & Not detected \\
& Post-flushing & Not detected \\
Hospital water harvesting tanks & Pre-flushing & Not detected \\
Collector pipes & Post-flushing & Not detected \\
& Pre-flushing & Not detected \\
Boiler & Post-flushing & $5 \times 10^{3}$ \\
& Pre-flushing & $4.5 \times 10^{3}$ \\
Room closest to the boiler (sink) & Post-flushing & $6.5 \times 10^{3}$ \\
Room farthest from the boiler (sink) & Pre-flushing & $7 \times 10^{3}$ \\
& Post-flushing & $4 \times 10^{3}$ \\
\hline
\end{tabular}

CFU/L: colony-forming units per liter. 
Table 3 Results of microbiological analysis from the hospital's hot water samples one day and one month after hyperchlorination

\begin{tabular}{|c|c|c|c|c|}
\hline \multicolumn{5}{|c|}{ Legionella pneumophila load, CFU/L } \\
\hline Sampling site & Sampling mode & Before treatment & One day after treatment & One month after treatment \\
\hline \multirow[t]{2}{*}{ Boiler } & Pre-flushing & $5 \times 10^{3}$ & $1.5 \times 10^{2}$ & $3.5 \times 10^{3}$ \\
\hline & Post-flushing & $4.5 \times 10^{3}$ & $2 \times 10^{2}$ & $5 \times 10^{3}$ \\
\hline \multirow[t]{2}{*}{ Room closest to the boiler (sink) } & Pre-flushing & $6.5 \times 10^{3}$ & $4 \times 10^{2}$ & $8.5 \times 10^{3}$ \\
\hline & Post- flushing & $7 \times 10^{3}$ & $2.5 \times 10^{3}$ & $6 \times 10^{3}$ \\
\hline \multirow{2}{*}{$\begin{array}{l}\text { Room farthest } \\
\text { from the boiler (sink) }\end{array}$} & Pre-flushing & $4 \times 10^{3}$ & $2.5 \times 10^{1}$ & $2.5 \times 10^{3}$ \\
\hline & Post-flushing & $5 \times 10^{2}$ & $1.5 \times 10^{2}$ & $3.5 \times 10^{2}$ \\
\hline \multirow{2}{*}{$\begin{array}{l}\text { Patient's room from } \\
\text { first admission (sink) }\end{array}$} & Pre-flushing & $4 \times 10^{3}$ & $2 \times 10^{2}$ & $2.5 \times 10^{3}$ \\
\hline & Post-flushing & $4.5 \times 10^{3}$ & $4 \times 10^{2}$ & $3 \times 10^{3}$ \\
\hline
\end{tabular}

CFU/L: colony-forming units per liter.

infiltrates. In addition, every time that the clinical features are equivocal, a negative result in urinary antigen tests should not be a reason for ruling out the disease.

Our patient was first empirically treated with clarithromycin to cover possible intracellular atypical respiratory pathogens [13]. After the isolation of the infectious agent, this treatment was not replaced with other antibiotics such as levofloxacin [14], because of the prompt clinical response to this macrolide and because of the previous poor response to a regimen with an antibiotic containing fluoroquinolone (ciprofloxacin). Indeed, the use of a fluoroquinole (ciprofloxacin) not specific for pulmonary infections and the short course (eight days) of treatment in a severely immunosuppressed patient could concur with the recurrence of respiratory symptoms and could have induced the third hospitalization.

Colonization of water systems by Legionella spp. occurs in hospitals throughout the world [15]. In hospital wards where immunosuppressed patients are subjected to chemotherapy drugs, hot water systems should be free of Legionella contamination.

The water system of our hospital was colonized by $L$. pneumophila of different serogroups. Although various methods to control Legionella in water distribution systems have been described in the literature (for example, methods based on physical or chemical treatments or both), none of these treatments is able to eradicate the bacterium permanently, and re-colonization occurs as soon as the treatments are interrupted [16]. Also, in our experience, the ineffectiveness of hyperchlorination treatments in eradicating L. pneumophila was demonstrated by the re-growth of Legionella only one month later to levels similar to those observed before treatment. This suggests a need for continuous monitoring and a maintenance regime for the hospital plumbing system, particularly in wards accommodating high-risk patients.

\section{Conclusions}

This case report demonstrates that (a) culture of respiratory secretions and real-time PCR for L. pneumophila should be part of the routine microbiological testing of BAL fluid from immunocompromised patients with pulmonary infiltrates, (b) a negative result from a urinary antigen test does not rule out the presence of legionellosis, and (c) the monitoring of the Legionella species in hospital water systems can help to bring in control measures to reduce the bacterial load and is therefore a proactive strategy for the control of the Legionella infection in hospitalized patients.

\section{Consent}

Written informed consent was obtained from the patient for publication of this case report and any accompanying images. A copy of the written consent is available for review by the Editor-in-Chief of this journal. The admitting hospital approved the use of patient samples and data.

\section{Abbreviations}

BAL: bronchoalveolar lavage; BCYE: buffered charcoal yeast extract; CFU: colony-forming units; CT: computed tomography; PCR: polymerase chain reaction.

\section{Acknowledgements}

This work was funded by Fondazione Cassa di Risparmio di Perugia, "Progetto Ricerca di Base". We are grateful to Maria Luisa Ricci, of the Department of Infectious, Parasitic and Immune-Mediated Diseases, Istituto Superiore di Sanità, Rome, Italy, for critical comments and suggestions and to Catherine Macpherson for editorial assistance.

\section{Author details}

${ }^{1}$ Department of Experimental Medicine and Biochemical Sciences, Microbiology Section, University of Perugia, Via del Giochetto, Perugia 06122 Italy. ${ }^{2}$ Department of Clinical and Experimental Medicine, Internal Medicine and Oncology Section, Santa Maria della Misericordia Hospital, Sant'Andrea delle Fratte, Perugia, Italy.

\section{Authors' contributions}

CC carried out the environmental investigation. AM performed the microbiological diagnosis and drafted the manuscript. AC and PF were responsible for the clinical management and therapy. AV drafted the 


\section{Competing interests}

The authors declare that they have no competing interests.

Received: 23 February 2011 Accepted: 17 August 2011

Published: 17 August 2011

\section{References}

1. Rota MC, Caporali MG, Giannitelli S, Mandarino G, Scaturro M, Ricci ML: Legionellosis in Italy in 2009: annual report [in Italian]. Annals of the Istituto Superiore di Sanità 2010, 23:3-10.

2. Leoni E, De Luca G, Legnani PP, Sacchetti R, Stampi S, Zanetti F: Legionella waterline colonization: detection of Legionella species in domestic, hotel and hospital hot water systems. J Appl Microbiol 2005, 98:373-379.

3. Kool JL, Bergmire-Sweat D, Butler JC, Brown EW, Peabody DJ, Massi DS, Carpenter JC, Pruckler JM, Benson RF, Fields BS: Hospital characteristics associated with colonization of water systems by Legionella and risk of nosocomial Legionnaires' disease: a cohort study of 15 hospitals. Infect Control Hosp Epidemiol 1999, 20:798-805.

4. Levin AS: Nosocomial legionellosis: prevention and management. Expert Rev Anti Infect Ther 2009, 7:57-68.

5. Fields BS, Benson RF, Besser RE: Legionella and Legionnaires' disease: 25 years of clinical investigation. Clin Microbiol Rev 2002, 15:506-526.

6. Oren I, Zuckerman T, Avivi I, Finkelstein R, Yigla M, Rowe JM: Nosocomial outbreak of Legionella pneumophila serogroup 3 pneumonia in a new bone marrow transplant unit: evaluation, treatment and control. Bone Marrow Transplantat 2002, 30:175-179.

7. Helbig JH, Bernander S, Castellani Pastoris M, Etienne J, Gaia V, Lauwers S, Lindsay D, Lück PC, Marques T, Mentula S, Peeters MF, Pelaz C, Struelens M, Uldum SA, Wewalka G, Harrison TG: Pan-European study on cultureproven Legionnaires' disease: distribution of Legionella pneumophila serogroups and monoclonal subgroups. Eur I Clin Microbiol Infect Dis 2002, 21:710-716.

8. Linee-guida recanti indicazioni sulla legionellosi per i gestori di strutture turistico-ricettive e termali. Gazzetta Ufficiale (Rome, Italy) 2005, $28: 54$.

9. Scaturro M, Losardo M, De Ponte G, Ricci ML: Comparison of three molecular methods used for subtyping of Legionella pneumophila strains isolated during an epidemic of Legionellosis in Rome. J Clin Microbiol 2005, 43:5348-5350.

10. Joseph C, Lee J, van Wijngaarden J, Drasar V, Castellani Pastoris M, of The European Surveillance Scheme for Travel Associated Legionnaires' Disease and the European Working Group for Legionella Infections: The European Guidelines for Control and Prevention of Travel Associated Legionnaires' Disease. London: Health Protection Agency; 2005 [http://www.hpa.org.uk/ web/HPAwebFile/HPAweb_C/1274093149925].

11. Kazandjian D, Chiew R, Gilbert GL: Rapid diagnosis of Legionella pneumophila serogroup 1 infection with the Binax enzyme immunoassay urinary antigen test. J Clin Microbiol 1997, 35:954-956.

12. Benson RF, Tang PW, Fields BS: Evaluation of the Binax and Biotest urinary antigen kits for detection of Legionnaires' disease due to multiple serogroups and species of Legionella. J Clin Microbiol 2000, 38:2763-2765.

13. Mandell LA: Etiologies of acute respiratory tract infections. Clin Infect Dis 2005, 41:503-506.

14. Mandell LA, lannini PB, Tillotson GS: Respiratory fluoroquinolones: differences in the details. Clin Infect Dis 2004, 38:1331-1332.

15. Liu WK, Healing DE, Yeomans JT, Elliot TS: Monitoring of hospital water supplies for Legionella. J Hosp Infect 1993, 24:1-9.

16. Hosein IK, Hill DW, Tan TY, Butchart EG, Wilson K, Finlay G, Burge S, Ribeiro CD: Point-of-care controls for nosocomial Legionellosis combined with chlorine dioxide potable water decontamination: a two-year survey at a Welsh teaching hospital. J Hosp Infect 2005, 61:100-106.

doi:10.1186/1752-1947-5-387

Cite this article as: Mencacci et al: Legionella pneumophila serogroup 3 pneumonia in a patient with low-grade 4 non-Hodgkin lymphoma: a case report. Journal of Medical Case Reports 2011 5:387.

\section{Submit your next manuscript to BioMed Central and take full advantage of:}

- Convenient online submission

- Thorough peer review

- No space constraints or color figure charges

- Immediate publication on acceptance

- Inclusion in PubMed, CAS, Scopus and Google Scholar

- Research which is freely available for redistribution

Submit your manuscript at www.biomedcentral.com/submit 